\title{
Graviton correlator and metric perturbations in de Sitter brane-world
}

\author{
Shin'ichi Nojiri* \\ Department of Applied Physics, National Defence Academy, Hashirimizu Yokosuka 239-8686, \\ JAPAN \\ Sergei D. Odintsov ${ }^{\dagger}$ \\ Instituto de Fisica de la Universidad de Guanajuato, Lomas del Bosque 103, Apdo. Postal E-143, \\ 37150 Leon, Gto., MEXICO \\ Sachiko Ogushi ${ }^{\ddagger}$ \\ Yukawa Institute for Theoretical Physics, Kyoto University, Kyoto 606-8502, JAPAN
}

\begin{abstract}
We consider de Sitter brane-world motivated by dS/CFT correspondence where both bulk and brane are de Sitter spaces. The brane tension is fixed by holographic RG. The $4 \mathrm{~d}$ effective action for metric perturbations and $4 \mathrm{~d}$ graviton correlator are explicitly found. The induced values of cosmological and Newton constants are calculated. The short distance behaviour of the graviton correlator (when no brane matter presents) turns out to be significally stronger than in the case of General Relativity. It is shown that quantum brane CFT gives the dominant contribution to graviton correlator on small scales like in Brane New World scenario.
\end{abstract}

98.80.Hw,04.50.+h,11.10.Kk,11.10.Wx

Typeset using REVTEX

\footnotetext{
*Electronic address: snojiri@yukawa.kyoto-u.ac.jp, nojiri@cc.nda.ac.jp

${ }^{\dagger}$ On leave from Tomsk State Pedagogical University, 634041 Tomsk, RUSSIA. Electronic address: odintsov@ifug5.ugto.mx

‡JSPS fellow, Electronic address: ogushi@yukawa.kyoto-u.ac.jp
} 


\section{INTRODUCTION}

The brane-world idea created the number of new and actively developing directions in cosmology. Especially, early time as well as quantum cosmology should be re-analyzed having in mind the possibility of large extra dimensions. Clearly, that is fundamental program which is still at the very beginning. In such situation (when already the big number of brane-world scenarios exists (see [1] for list of references)) only some particular results in frames of specific models may be considered.

Among the existing inflationary brane-world scenarios there is one quantum brane-world (or Brane New World) [2,3] scenario where quantum effects play the dominant role. This (AdS bulk) model is formulated in terms of AdS/CFT correspondence, so classical brane tension is fixed from the very beginning. CFT living on the brane provides the effective tension (via the account of corresponding conformal anomaly), giving the possibility of fourdimensional brane inflation. The careful calculation of graviton correlator and perturbations in Brane New World leads to quite remarkable result [3]: CFT strongly suppresses metric perturbations at small scale (microwave sky). It is expected that this is quite universal phenomenon in quantum cosmology.

It is extremely interesting to check the universality of this phenomenon in other braneworlds. In particular, with recently proposed dS/CFT correspondence (see ref. [4] for introduction) there may be necessary to study brane-worlds with de Sitter bulk. One such model formulated in the analogy with Brane New World was suggested in ref. [5]. Even in situation when surface counterterms (classical brane tension) are fixed (by requirement of finiteness of de Sitter bulk or by holographic RG considerations) one finds the existence of de Sitter brane without the introduction of effective tension by brane quantum fields [5]. Unlike to Brane New World model with AdS bulk, an arbitrary CFT may be considered on the brane, the classical dS brane solution will be just modified by quantum effects.

It is the purpose of the present work to investigate the metric perturbations and graviton correlator in 5d de Sitter brane-world scenario [5] using methods developed in ref. [3]. In the next section the formulation of dS bulk model with dS brane on it is presented. The effective action for metric perturbations as well as $4 \mathrm{~d}$ graviton correlator are found. The induced values of $4 \mathrm{~d}$ cosmological and gravitational constants in terms of $5 \mathrm{~d}$ quantities are calculated. It is shown that short distance behaviour of the correlator is similar to that from $5 \mathrm{~d}$ AdS bulk (with $4 \mathrm{~d}$ inflationary brane). In other words, the induced gravity force is getting stronger than the one in General Relativity at short distances. It is observed that when brane tension is free parameter it is easiear to realize the inflationary brane in dS bulk. The graviton correlator is found also in this case when bulk has Lorentzian signature. The possibility to have brane radius bigger than length parameter of dS bulk is observed.

Section three is devoted to account of brane CFT effects to graviton correlator. Using conformal anomaly the CFT action for metric perturbations is found. It is shown that CFT gives the dominant contribution to graviton correlator at short disctances, similarly to the case with AdS bulk. Some outlook is given in the Discussion. 


\section{GRAVITON CORRELATOR IN DE SITTER BRANE-WORLD}

We start from the action $S$ which is the sum of the Einstein-Hilbert action $S_{\mathrm{EH}}$ with positive cosmological constant, the Gibbons-Hawking surface term $S_{\mathrm{GH}}$ and the first counter term $S_{1}$ :

$$
\begin{aligned}
S & =S_{\mathrm{EH}}+S_{\mathrm{GH}}+S_{1}, \quad S_{\mathrm{EH}}=-\frac{1}{16 \pi G} \int d^{5} x \sqrt{g}\left(R-\frac{12}{l^{2}}\right), \\
S_{\mathrm{GH}} & =-\frac{1}{8 \pi G} \int d^{4} x \sqrt{\gamma} K, \quad S_{1}=\frac{3}{8 \pi G l} \int d^{4} x \sqrt{\gamma} .
\end{aligned}
$$

Here the 5 -dimensional bulk spacetime metric is given by the $g_{\mu \nu}$ and the boundary 4dimensional spacetime metric is given by $\gamma_{i j} . K$ is the trace of the extrinsic curvature of the boundary, which is defined as

$$
K_{\mu \nu}=\xi_{\mu}^{\rho} \xi_{\nu}^{\sigma} \nabla_{\rho} n_{\sigma}, \quad \xi_{\mu}^{\nu}=\delta_{\mu}^{\nu}-n_{\mu} n^{\nu},
$$

where Greek indices are five dimensional and $n_{\mu}$ is the unit vector normal to the boundary: $n_{\mu}=(l, 0,0,0,0)$. The coefficient of $S_{1}$ can be determined from the condition of the finiteness of the action (but in a less strict way than in AdS/CFT). Moreover, using the holographic renormailzation group method [6] this coefficient can be determined uniquely [0].

We consider 5-dimensional Euclidean de Sitter space which is identical with 5-dimensional sphere $S^{5}$ :

$$
\begin{aligned}
d s_{S_{5}}^{2} & =l^{2}\left(d y^{2}+\gamma_{i j} d x^{i} d x^{j}\right) \\
\gamma_{i j} & =\sin ^{2} y \hat{\gamma}_{i j},
\end{aligned}
$$

where $\hat{\gamma_{i j}} d x^{i} d x^{j}$ describes the metric of $\mathrm{S}_{4}$ with unit radius.

The metric of $S_{4}$ with the unit radius is given by

$$
d \Omega_{4}^{2}=d \chi^{2}+\sin ^{2} \chi d \Omega_{3}^{2} .
$$

Here $d \Omega_{3}^{2}$ is described by the metric of 3 dimensional unit sphere. If one changes the coordinate $\chi$ to $\sigma$ by $\sin \chi= \pm \frac{1}{\cosh \sigma}$, one obtainst

$$
d \Omega_{4}^{2}=\frac{1}{\cosh ^{2} \sigma}\left(d \sigma^{2}+d \Omega_{3}^{2}\right) .
$$

Now one assumes the metric of 5 dimensional spacetime as follows:

\footnotetext{
${ }^{1}$ We adopt the conventions from ref. [3]

${ }^{2}$ If we Wick-rotate the metric by $\sigma \rightarrow i t$, we obtain the metric of de Sitter space:

$$
d \Omega_{4}^{2} \rightarrow d s_{\mathrm{dS}}^{2}=\frac{1}{\cos ^{2} t}\left(-d t^{2}+d \Omega_{3}^{2}\right)
$$
}




$$
d s^{2}=l^{2}\left(d y^{2}+\mathrm{e}^{2 A(y, \sigma)} \tilde{\gamma}_{i j} d x^{i} d x^{j}\right), \quad \tilde{\gamma}_{i j} d x^{i} d x^{j} \equiv d \sigma^{2}+d \Omega_{3}^{2}
$$

Due to the assumption (6), the actions (11) have the following forms:

$$
\begin{aligned}
S_{\mathrm{EH}} & =-\frac{l^{3} V_{3}}{16 \pi G} \int d y d \sigma\left\{\left(-8 \partial_{y}^{2} A-20\left(\partial_{y} A\right)^{2}\right) \frac{\mathrm{e}^{4 A}}{l^{2}}\right. \\
& \left.+\frac{1}{l^{2}}\left(-6 \partial_{\sigma}^{2} A-6\left(\partial_{\sigma} A\right)^{2}+6\right) \mathrm{e}^{2 A}-\frac{12}{l^{2}} \mathrm{e}^{4 A}\right\} \\
S_{\mathrm{GH}} & =-\frac{l^{3} V_{3}}{2 \pi G} \int d \sigma \mathrm{e}^{4 A} \partial_{y} A, \quad S_{1}=\frac{3 l^{3} V_{3}}{8 \pi G} \int d \sigma \mathrm{e}^{4 A} .
\end{aligned}
$$

Here $V_{3}$ is the volume or area of the unit 3 sphere and it is assumed that there is a brane at $y=$ constant.

In the bulk, one obtains the following equation of motion from $S_{\mathrm{EH}}$ by the variation over A:

$$
0=\left(-24 \partial_{y}^{2} A-48\left(\partial_{y} A\right)^{2}-48\right) \mathrm{e}^{4 A}+\left(-12 \partial_{\sigma}^{2} A-12\left(\partial_{\sigma} A\right)^{2}+12\right) \mathrm{e}^{2 A}
$$

which corresponds to one of the Einstein equations. Then one finds solutions, $A_{S}$, which correspond to the metric $\mathrm{dS}_{5}$ :

$$
A=A_{S}=\ln \sin y-\ln \cosh \sigma .
$$

On the brane at the boundary, one gets the following equation:

$$
0=\frac{48 l^{3}}{16 \pi G}\left(\partial_{y} A-1\right) \mathrm{e}^{4 A}
$$

Substituting the bulk solution $A=A_{S}$ in (9) into (10) and defining the radius $R_{b}$ of the brane by $R_{b} \equiv l \sin y_{0}$, one obtains

$$
0=\frac{1}{\pi G}\left(\frac{1}{R_{b}} \sqrt{1-\frac{R_{b}^{2}}{l^{2}}}-\frac{1}{l}\right) R_{b}^{4} .
$$

First we should note $0 \leq R_{b} \leq l$ by definition. Eq.(11) has a solution [5]:

$$
R_{b}^{2}=R_{b 0}^{2} \equiv \frac{l^{2}}{2} \text { or } y_{0}=\frac{\pi}{4}, \frac{3 \pi}{4} .
$$

In Eq.(11), the first term $\frac{R_{b}^{3}}{\pi G} \sqrt{1-\frac{R_{b}^{2}}{l^{2}}}$ corresponds to the gravity, which makes the radius $R_{b}$ larger. On the other hand, the second term $-\frac{R_{b}^{4}}{\pi G l}$ corresponds to the tension, which makes $R_{b}$ smaller. When $R_{b}<R_{b 0}$, gravity becomes larger than the tension and vice versa when $R_{b}>R_{b 0}$. Then both of the solutions (12) are stable. Although it is not clear from (11), $R_{b}=l\left(y=\frac{\pi}{2}\right)$ corresponds to the local maximum. This is because the point $y=\frac{\pi}{2}$ is a symmetric point. At the point, since the strength of gravitational forces from two bulk spaces is equal and their signs are different with each other, the gravitaional forces to the brane are balanced. Furthermore, since $R_{b}$ becomes maximum there, the energy coming 
from the tension of the brane is maximum. Then the point $y=\frac{\pi}{2}$ becomes a local maximum for both of the gravitational force and tension of the brane. To see the above situation more, clearly we consider an effective potential $V(y)$. Since Eq.(10) are obtained from the variation of $A$, maybe we can define $V(y)$ as follows,

$$
\begin{aligned}
V(y) & =\left.\frac{1}{\pi G} \int\left(\frac{1}{R_{b}} \sqrt{1-\frac{R_{b}^{2}}{l^{2}}}-\frac{1}{l}\right) R_{b}^{4} d A\right|_{\sigma: \text { fixed }} \\
& =\frac{l^{3}}{\pi G} \int\left(\frac{|\cos y|}{\sin y}-1\right) \sin ^{4} y \cdot \frac{\cos y}{\sin y} d y \\
& =V_{0}+\frac{l^{3}}{\pi G}\left\{\frac{1}{8}\left(\sin y-2 \sin ^{3} y\right)|\cos y|+\frac{1}{8}\left|y-\frac{\pi}{2}\right|+\frac{1}{4} \sin ^{4} y\right\} .
\end{aligned}
$$

Here we used Eq.(9) and $V_{0}$ is a constant of the integration. Then we find

$$
\begin{aligned}
& V(0)-V_{0}=V(\pi)-V_{0}=\frac{l^{3}}{\pi G} \frac{\pi}{16}, \quad V\left(\frac{\pi}{4}\right)-V_{0}=V\left(\frac{3 \pi}{4}\right)-V_{0}=\frac{l^{3}}{\pi G}\left(\frac{1}{16}-\frac{\pi}{32}\right), \\
& V\left(\frac{\pi}{2}\right)-V_{0}=\frac{l^{3}}{\pi G} \frac{1}{4}, \quad V^{\prime}(0)=V^{\prime}\left(\frac{\pi}{4}\right)=V^{\prime}\left(\frac{\pi}{2}\right)=V^{\prime}\left(\frac{3 \pi}{4}\right)=V^{\prime}(\pi)=0
\end{aligned}
$$

The conceptual (not exact) shape of the potential is given in Fig.11. From the figure, we can find that there are local maxima at $y=0, \frac{\pi}{2}$ and $\pi$ and minima at $y=\frac{\pi}{4}$ and $\frac{3 \pi}{4}$. 


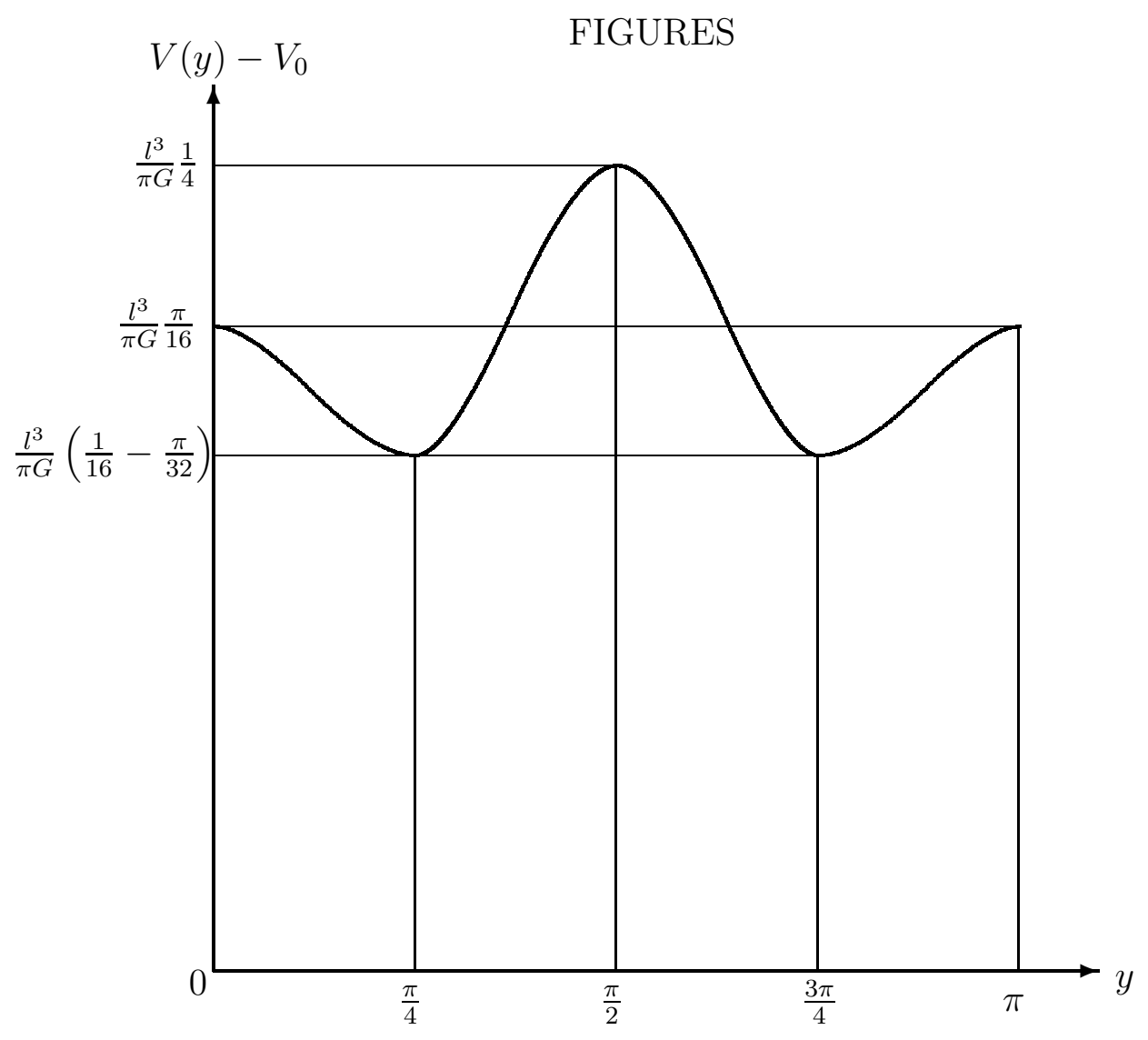

FIG. 1. Conceptual shape of the effective potential $V(y)$ with respect to the position of the brane $y$.

Hence, the possibility of creation of inflationary brane in de Sitter bulk is possible already on classical level, even in situation when brane tension is fixed by holographic RG. That is qualitatively different from the case of AdS bulk where only quantum effects led to creation of inflationary brane [2, 3] (when brane tension was not free parameter).

It is interesting to study the effect of the perturbations of the gravity on the sphere. In case when bulk is AdS space it has been examined in ref. [3] with the help of the AdS/CFT correspondence. We also study it applying the considerations from the proposed dS/CFT correspondence (for the introduction, see 四). Then we rewrite bulk metric which includes the effect of the perturbation $h_{i j}(y, x)$ as follows:

$$
d s_{\mathrm{S}_{5}}^{2}=l^{2} d y^{2}+\left(l^{2} \sin ^{2} y \hat{\gamma}_{i j}+h_{i j}(y, x)\right) d x^{i} d x^{j} .
$$

$h_{i j}$ is transverse and traceless with respect to the metric on the spherical spatial sections:

$$
\hat{\gamma}^{i j} h_{i j}(y, x)=\hat{\nabla}^{i} h_{i j}(y, x)=0,
$$

with $\hat{\nabla}$ being the covariant derivative defined by the metric $\hat{\gamma}_{i j}$. We use the Greek letters $\mu, \nu, \cdots$ for 5 dimensional vector indeces and the Roman letters $i, j, \cdots$ for 4 dimensional indeces. The Einstein equation in the bulk leads to the classical solutions of scalar curvature $R$, Ricci tensor $R_{\mu \nu}$ and Riemann tensor $R_{\mu \rho \nu \sigma}$ as follows: 


$$
\begin{aligned}
R & =\frac{D}{D-2} \Lambda, \quad R_{\mu \nu}=\frac{\Lambda}{D-2} g_{\mu \nu}, \\
R_{\mu \rho \nu \sigma} & =\frac{\Lambda}{(D-1)(D-2)}\left(g_{\mu \nu} g_{\rho \sigma}-g_{\mu \sigma} g_{\rho \nu}\right) .
\end{aligned}
$$

Here $D$ and $\Lambda$ is the bulk dimension and cosmological constant respectively. We take $D=5$ and $\Lambda$ is related with the radius of $S_{5}$ by $\Lambda=\frac{12}{l^{2}}$. In the background of $S_{4}$, the linearized Einstein equation has the following form:

$$
\nabla^{2} h_{\mu \nu}=\frac{2}{l^{2}} h_{\mu \nu} .
$$

We now use the standard tensor spherical harmonics $H_{i j}^{(p)}(x)$ to expand the metric perturbation

$$
\hat{\gamma}^{i j} H_{i j}^{(p)}(x)=\hat{\nabla}^{i} H_{i j}^{(p)}(x)=0,
$$

They are tensor eigenfunctions of the Laplacian:

$$
\hat{\nabla}^{2} H_{i j}^{(p)}=(2-p(p+d-1)) H_{i j}^{(p)},
$$

where $p=2,3, \cdots$, and $d$ is the dimension of the boundary, $D=d+1$. As usual in the quantum mechanics, the eigenvalue of $-\nabla^{2}$ can be regarded as the square of the momentum. Especially when $p$ is large, we can regard $p$ as the absolute value of the momentum. The metric perturbation can be written as a sum of separable perturbations of the form

$$
h_{i j}(y, x)=f_{p}(y) H_{i j}^{(p)}(x) .
$$

Substituting this into (18), we obtain the following differential equation:

$$
\begin{aligned}
& f_{p}^{\prime \prime}(y)+(d-4) \cot y f_{p}^{\prime}(y) \\
& -\left\{-2(d-2)+[p(p+d-1)+2(d-3)] \sin ^{-2} y\right\} f_{p}(y)=0 .
\end{aligned}
$$

Here ' denotes the derivative with respect to $y$. The solution can be written by associated Legendre functions:

$$
f_{p}(y) \propto(\sin y)^{(5-d) / 2} P_{-(d+1) / 2}^{-(p+(d-1) / 2)}(\cos y) .
$$

The full solution for the metric perturbation is

$$
h_{i j}(y, x)=\sum_{p} \frac{f_{p}(y)}{f_{p}\left(y_{0}\right)} H_{i j}^{p}(x) \int d^{4} x^{\prime} \sqrt{\hat{\gamma}} h^{k l}\left(x^{\prime}\right) H_{k l}^{(p)}\left(x^{\prime}\right) .
$$

Here $h^{k l}(x)$ is the boundary value of the metric perturbation. This discussion generalizes the study of ref. [3] done in AdS bulk to dS bulk.

By using equations (16), (17), (18), one can obtain the explicit form of the action (11) from the perturbated metric (15). Then the Einstein-Hilbert action with the perturbations is given by 


$$
\begin{aligned}
S_{\mathrm{EH}}= & -\frac{1}{16 \pi G} \int d^{5} x \sqrt{g}\left(R-\frac{12}{l^{2}}\right) \\
= & -\frac{1}{16 \pi G} \int d^{5} x \sqrt{g}\left(\frac{8}{l^{2}}+\frac{1}{4} h^{\mu \nu} \nabla^{2} h_{\mu \nu}-\frac{1}{2 l^{2}} h^{\mu \nu} h_{\mu \nu}\right), \\
& -\frac{1}{16 \pi G} \int d^{4} x \sqrt{\gamma}\left(-\frac{1}{2} n^{\mu} h^{\nu \rho} \nabla_{\nu} h_{\mu \rho}+\frac{3}{4} h_{\nu \rho} n^{\mu} \nabla_{\mu} h^{\nu \rho}\right) .
\end{aligned}
$$

Since the bulk part of the action always vanishes, the action is to be expressed in terms of the boundary values of the perturbation $h_{i j}$ :

$$
\begin{aligned}
S_{\mathrm{EH}}= & -\frac{l^{3}}{2 \pi G} \int d^{4} x \sqrt{\hat{\gamma}} \int_{0}^{y_{0}} d y \sin ^{4} y \\
& -\frac{l^{3}}{16 \pi G} \int d^{4} x \sqrt{\hat{\gamma}}\left(\frac{3}{4 l^{4}} h^{i j} \partial_{y} h_{i j}-\frac{\cot y_{0}}{l^{4}} h^{i j} h_{i j}\right),
\end{aligned}
$$

where we are now raising and lowering the indeces $i, j, \cdots$ by using $\hat{\gamma}_{i j}$ as in ref. [3. The Gibbons-Hawking term has the following form:

$$
\begin{aligned}
S_{\mathrm{GH}} & =-\frac{1}{8 \pi G} \int d^{4} x \sqrt{\gamma} K \\
& =-\frac{l^{3}}{2 \pi G} \int d^{4} x \sqrt{\hat{\gamma}}\left(\sin ^{3} y_{0} \cos y_{0}-\frac{1}{8 l^{4}} h^{i j} \partial_{y} h_{i j}\right) .
\end{aligned}
$$

The counter term $S_{1}$ is

$$
\begin{aligned}
S_{1} & =\frac{3}{8 \pi G l} \int d^{4} x \sqrt{\gamma}, \\
& =\frac{3 l^{3}}{8 \pi G} \int d^{4} x \sqrt{\hat{\gamma}}\left(\sin ^{4} y_{0}-\frac{1}{4 l^{4}} h^{i j} h_{i j}\right) .
\end{aligned}
$$

Then the total action becomes

$$
\begin{aligned}
S= & S_{\mathrm{EH}}+S_{\mathrm{GH}}+S_{1}, \\
= & \frac{l^{3}}{2 \pi G} \int d^{4} x \sqrt{\hat{\gamma}}\left\{-\frac{3}{4} \sin ^{3} y_{0} \cos y_{0}-\frac{3}{8} y_{0}+\frac{3}{8} \cos y_{0} \sin y_{0}\right. \\
& \left.+\frac{1}{32 l^{4}} h^{i j} \partial_{y} h_{i j}+\frac{1}{16 l^{4}}\left(-3+2 \cot y_{0}\right) h^{i j} h_{i j}+\frac{3}{4} \sin ^{4} y_{0}\right\} .
\end{aligned}
$$

Eq.(29) describes the fluctuation of $\mathrm{S}_{4}$ brane in the bulk $\mathrm{S}_{5}$, or after Wick-rotation, 3 dimensional dS brane in the bulk 5-dimensional dS space.

In case that the bulk space is 5 dimensional Euclidean anti-de Sitter space (hyperboloid), if we choose the metric as

$$
\begin{aligned}
d s_{\mathrm{S}_{5}}^{2} & =l^{2}\left(d y^{2}+\gamma_{i j} d x^{i} d x^{j}\right) \\
\gamma_{i j} & =\sinh ^{2} y \hat{\gamma}_{i j}
\end{aligned}
$$

instead of (3) and assume that the brane lies at $y=y_{0}$, the equation corresponding to (29 has the following form 


$$
\begin{aligned}
S^{\text {AdS }}= & \frac{l^{3}}{2 \pi G} \int d^{4} x \sqrt{\hat{\gamma}}\left\{-\frac{3}{4} \sinh ^{3} y_{0} \cosh y_{0}-\frac{3}{8} y_{0}+\frac{3}{8} \cosh y_{0} \sinh y_{0}\right. \\
& \left.+\frac{1}{32 l^{4}} h^{i j} \partial_{y} h_{i j}+\frac{1}{16 l^{4}}\left(-3+2 \operatorname{coth} y_{0}\right) h^{i j} h_{i j}+\frac{3}{4} \sinh ^{4} y_{0}\right\}
\end{aligned}
$$

The expression (31) can be also obtained from (29) by replacing sin and cos by sinh and cosh, respectively. In fact, the function $f_{p}$ corresponding to (23) is also given by [a],

$$
f_{p}^{\operatorname{AdS}}(y) \propto(\sinh y)^{(5-d) / 2} P_{-(d+1) / 2}^{-(p+(d-1) / 2)}(\cosh y) .
$$

Now one can consider the Euclidean graviton correlator, $\left\langle h_{i j}(x) h_{i^{\prime} j^{\prime}}\left(x^{\prime}\right)\right\rangle$ (for earlier study on four-dimensional graviton correlator in maximally symmetric spaces, see [《] and references therein). To read off graviton correlator from eq.(29), we focus on the $h^{i j} \partial_{y} h_{i j}$ and $h^{i j} h_{i j}$ terms. The expansion of $\partial_{y} h_{i j}$ at $y=y_{0}$ is obtained by using (24) as

$$
\partial_{y} h_{i j}=\sum_{p} \frac{f_{p}^{\prime}\left(y_{0}\right)}{f_{p}\left(y_{0}\right)} H_{i j}^{p}(x) \int d^{4} x^{\prime} \sqrt{\hat{\gamma}} h^{k l}\left(x^{\prime}\right) H_{k l}^{(p)}\left(x^{\prime}\right) .
$$

The terms related with the graviton correlator in the action (29) can be rewritten as

$$
S_{\text {correlator }}=\sum_{p}\left(\int d^{4} x^{\prime} \sqrt{\hat{\gamma}} h^{k l}\left(x^{\prime}\right) H_{k l}^{(p)}\left(x^{\prime}\right)\right)^{2} T_{p}\left(y_{0}\right),
$$

where

$$
T_{p}\left(y_{0}\right)=\frac{l^{3}}{2 \pi G} \frac{1}{32 l^{4}}\left(\frac{f_{p}^{\prime}\left(y_{0}\right)}{f_{p}\left(y_{0}\right)}+4 \cot y_{0}-6\right) .
$$

With above equations one arrives at the graviton correlator as

$$
\begin{aligned}
\left\langle h_{i j}(x) h_{i^{\prime} j^{\prime}}\left(x^{\prime}\right)\right\rangle & =\sum_{p=2}^{\infty} W_{i j i^{\prime} j^{\prime}}^{(p)}\left(x, x^{\prime}\right) \frac{1}{2 T_{p}\left(y_{0}\right)}, \\
W_{i j i^{\prime} j^{\prime}}^{(p)}\left(x, x^{\prime}\right) & \equiv H_{i j}^{(p)}(x) H_{i^{\prime} j^{\prime}}^{(p)}\left(x^{\prime}\right) .
\end{aligned}
$$

Using (23), we can rewrite (35) for $d=4$ as following form,

$$
T_{p}\left(y_{0}\right)=\frac{1}{64 \pi G l}\left\{-6-(p-3) \cot y_{0}-\frac{P_{-5 / 2}^{-p-1 / 2}\left(\cos y_{0}\right)}{P_{-5 / 2}^{-p-3 / 2}\left(\cos y_{0}\right)}\right\} .
$$

For our solutions $y_{0}=\frac{\pi}{4}, \frac{3 \pi}{4}$ (12) $), T_{p}\left(y_{0}\right)$ becomes

$$
\begin{aligned}
T_{p}\left(\frac{\pi}{4}\right) & =\frac{1}{64 \pi G l}\left\{-p-3-\frac{P_{-5 / 2}^{-p-1 / 2}\left(\frac{1}{\sqrt{2}}\right)}{P_{-5 / 2}^{-p-3 / 2}\left(\frac{1}{\sqrt{2}}\right)}\right\}, \\
T_{p}\left(\frac{3 \pi}{4}\right) & =\frac{1}{64 \pi G l}\left\{p-9-\frac{P_{-5 / 2}^{-p-1 / 2}\left(-\frac{1}{\sqrt{2}}\right)}{P_{-5 / 2}^{-p-3 / 2}\left(-\frac{1}{\sqrt{2}}\right)}\right\} .
\end{aligned}
$$


Now one can consider the perturbation based on 4-dimensional action, in order to compare it with that of the 4-dimensional gravity induced from the bulk 5-dimensional dS gravity. We now introduce 4-dimensional cosmological constant $\Lambda^{(4)}$ and the Newton constant $G^{(4)}$, which would be adjusted later to be consistent with the induced gravity. The perturbed 4-dimensional Einstein-Hilbert action is given as follows:

$$
\begin{aligned}
S_{\mathrm{EH}}^{(4)} & =-\frac{1}{16 \pi G^{(4)}} \int d^{4} x \sqrt{g}\left(R+\Lambda^{(4)}\right), \\
& =S_{0}^{(4)}-\frac{1}{16 \pi G^{(4)}} \int d^{4} x \sqrt{g}\left\{\frac{1}{4} h^{i j} \hat{\nabla}^{2} h_{i j}+\left(-\frac{1}{4} \Lambda^{(4)}-\frac{2}{l^{2} \sin ^{2} y_{0}}\right) h^{i j} h_{i j}\right\} .
\end{aligned}
$$

Using eq.(20), we can obtain the function for calculating graviton correlator (instead of (35)) as

$$
T_{p}^{(4)}\left(y_{0}\right)=-\frac{1}{16 \pi G^{(4)}}\left\{\frac{1}{4 l^{2} \sin ^{2} y_{0}}(2-p(p+3))-\frac{1}{4} \Lambda^{(4)}-\frac{2}{l^{2} \sin ^{2} y_{0}}\right\} .
$$

Eq. (20) tells that $p(=2,3, \cdots)$ is proportional to the magnitude of the momentum of the graviton. In the analogy with the Randall-Sundrum model [9], we expect the present model of the 4 dimensional gravity induced from the 5 dimensional bulk gravity would reproduce the 4-dimensional Einstein gravity in the low-energy limit. Then we compare (38) and (40) for $p=2,3$ cases and determine $G^{(4)}$ and $\Lambda^{(4)}$ so that the two expressions coincide with each other. First we consider $y_{0}=\frac{\pi}{4}$ solution. Then one has

$$
\frac{1}{64 \pi G l}\left(-5-\frac{P_{-\frac{5}{2}}^{-\frac{5}{2}}\left(\frac{1}{\sqrt{2}}\right)}{P_{-\frac{5}{2}}^{-\frac{7}{2}}\left(\frac{1}{\sqrt{2}}\right)}\right)=-\frac{1}{16 \pi G^{(4)}}\left(-\frac{8}{l^{2}}-\frac{\Lambda^{(4)}}{4}\right)
$$

for $p=2$ and

$$
\frac{1}{64 \pi G l}\left(-6-\frac{P_{-\frac{5}{2}}^{-\frac{7}{2}}\left(\frac{1}{\sqrt{2}}\right)}{P_{-\frac{5}{2}}^{-\frac{9}{2}}\left(\frac{1}{\sqrt{2}}\right)}\right)=-\frac{1}{16 \pi G^{(4)}}\left(-\frac{12}{l^{2}}-\frac{\Lambda^{(4)}}{4}\right)
$$

for $p=3$. As a result

$$
\begin{gathered}
\Lambda^{(4)}=\frac{16}{l^{2}} \frac{3+3 \frac{P_{-\frac{5}{2}}^{-\frac{5}{2}}\left(\frac{1}{\sqrt{2}}\right)}{P_{-\frac{5}{2}}^{-\frac{7}{2}}\left(\frac{1}{\sqrt{2}}\right)}-2 \frac{P_{-\frac{5}{2}}^{-\frac{7}{2}}\left(\frac{1}{\sqrt{2}}\right)}{P_{-\frac{5}{2}}^{-\frac{9}{2}}\left(\frac{1}{\sqrt{2}}\right)}}{1-\frac{P_{-\frac{5}{2}}^{-\frac{5}{2}}\left(\frac{1}{\sqrt{2}}\right)}{P_{-\frac{5}{2}}^{-\frac{7}{2}}\left(\frac{1}{\sqrt{2}}\right)}+\frac{P_{-\frac{5}{2}}^{-\frac{7}{2}}\left(\frac{1}{\sqrt{2}}\right)}{P_{-\frac{5}{2}}^{-\frac{9}{2}}\left(\frac{1}{\sqrt{2}}\right)}} \\
\frac{1}{16 \pi G^{(4)}}=-\frac{l}{256 \pi G}\left(1-\frac{P_{-\frac{5}{2}}^{-\frac{5}{2}}\left(\frac{1}{\sqrt{2}}\right)}{P_{-\frac{5}{2}}^{-\frac{7}{2}}\left(\frac{1}{\sqrt{2}}\right)}+\frac{P_{-\frac{5}{2}}^{-\frac{7}{2}}\left(\frac{1}{\sqrt{2}}\right)}{P_{-\frac{5}{2}}^{-\frac{9}{2}}\left(\frac{1}{\sqrt{2}}\right)}\right) .
\end{gathered}
$$


This gives the $4 \mathrm{~d}$ parameters $G^{(4)}$ and $\Lambda^{(4)}$ in terms of the $5 \mathrm{~d}$ parameters so that $T_{p}\left(\frac{\pi}{4}\right)$ coincides with $T_{p}^{(4)}$ for $p=2$ and 3, which corresponds to the infrared behavior. Of course, there will appear some difference for higher $p=4,5,6, \cdots$. As we will see later, with account of the quantum effects from the matter fields on the brane, the difference between $T_{p}\left(\frac{\pi}{4}\right)$ and $T_{p}^{(4)}$ disappears in the ultraviolet region. Then both of infrared and ultraviolet behaviors can be reproduced from the induced gravity.

On the other hand, for the solution $y_{0}=\frac{3 \pi}{4}$, one gets

$$
\frac{1}{64 \pi G l}\left(-7-\frac{P_{-\frac{5}{2}}^{-\frac{5}{2}}\left(-\frac{1}{\sqrt{2}}\right)}{P_{-\frac{5}{2}}^{-\frac{7}{2}}\left(-\frac{1}{\sqrt{2}}\right)}\right)=-\frac{1}{16 \pi G^{(4)}}\left(-\frac{8}{l^{2}}-\frac{\Lambda^{(4)}}{4}\right)
$$

for $p=2$ and

$$
\frac{1}{64 \pi G l}\left(-6-\frac{P_{-\frac{5}{2}}^{-\frac{7}{2}}\left(-\frac{1}{\sqrt{2}}\right)}{P_{-\frac{5}{2}}^{-\frac{9}{2}}\left(-\frac{1}{\sqrt{2}}\right)}\right)=-\frac{1}{16 \pi G^{(4)}}\left(-\frac{12}{l^{2}}-\frac{\Lambda^{(4)}}{4}\right)
$$

for $p=3$. Then

$$
\begin{gathered}
\Lambda^{(4)}=-\frac{16}{l^{2}} \frac{9+3 \frac{P_{-\frac{5}{5}}^{-\frac{5}{5}}\left(-\frac{1}{\sqrt{2}}\right)}{P_{-\frac{5}{2}}^{-\frac{7}{2}}\left(-\frac{1}{\sqrt{2}}\right)}-2 \frac{P_{-\frac{7}{5}}^{-\frac{7}{5}}\left(-\frac{1}{\sqrt{2}}\right)}{P_{-\frac{5}{2}}^{-\frac{9}{2}}\left(-\frac{1}{\sqrt{2}}\right)}}{1+\frac{P_{-\frac{5}{2}}^{-\frac{5}{2}}\left(-\frac{1}{\sqrt{2}}\right)}{P_{-\frac{5}{2}}^{-\frac{7}{2}}\left(-\frac{1}{\sqrt{2}}\right)}-\frac{P_{-\frac{5}{2}}^{-\frac{7}{2}}\left(-\frac{1}{\sqrt{2}}\right)}{P_{-\frac{5}{2}}^{-\frac{9}{2}}\left(-\frac{1}{\sqrt{2}}\right)}} \\
\frac{1}{16 \pi G^{(4)}}=\frac{l}{256 \pi G}\left(1+\frac{P_{-\frac{5}{2}}^{-\frac{5}{2}}\left(-\frac{1}{\sqrt{2}}\right)}{P_{-\frac{5}{2}}^{-\frac{7}{2}}\left(\frac{-1}{\sqrt{2}}\right)}-\frac{P_{-\frac{5}{2}}^{-\frac{7}{2}}\left(-\frac{1}{\sqrt{2}}\right)}{P_{-\frac{5}{2}}^{-\frac{9}{2}}\left(-\frac{1}{\sqrt{2}}\right)}\right) .
\end{gathered}
$$

Again, the effective Newton constant and the effective cosmological constant of the induced 4-dimensional gravity are expressed in terms of the bulk 5-dimensional gravity parameters.

We now investigate the short distance behaviour, which is determined from the large $p$ behavior. When $p$ is large

$$
P_{-\frac{5}{2}}^{-p-\frac{1}{2}}\left(\cos y_{0}\right) \rightarrow \frac{\mathrm{e}^{\left(-p-\frac{1}{2}\right) \pi i}}{\Gamma\left(\frac{3}{2}+p\right)}\left(\frac{1+\cos y_{0}}{1-\cos y_{0}}\right)^{-\frac{p+\frac{1}{2}}{2}} .
$$

Then one obtains

$$
\frac{P_{-\frac{5}{2}}^{-p-\frac{1}{2}}\left(\cos y_{0}\right)}{P_{-\frac{5}{2}}^{-p-\frac{3}{2}}\left(\cos y_{0}\right)} \rightarrow-\left(\frac{3}{2}+p\right) \sqrt{\frac{1+\cos y_{0}}{1-\cos y_{0}}} .
$$

Especially for the solution $y_{0}=\frac{\pi}{4}$, we find 


$$
\frac{P_{-\frac{5}{2}}^{-p-\frac{1}{2}}\left(\frac{1}{\sqrt{2}}\right)}{P_{-\frac{5}{2}}^{-p-\frac{3}{2}}\left(\frac{1}{\sqrt{2}}\right)} \rightarrow-\left(\frac{3}{2}+p\right)(\sqrt{2}+1)
$$

and for the solution $y_{0}=\frac{3 \pi}{4}$, one gets

$$
\frac{P_{-\frac{5}{2}}^{-p-\frac{1}{2}}\left(-\frac{1}{\sqrt{2}}\right)}{P_{-\frac{5}{2}}^{-p-\frac{3}{2}}\left(-\frac{1}{\sqrt{2}}\right)} \rightarrow-\left(\frac{3}{2}+p\right)(\sqrt{2}-1) .
$$

Then $T_{p}(38)$ is linear for large $p$ :

$$
T_{p}\left(\frac{\pi}{4}\right) \rightarrow-\frac{(\sqrt{2}+2) p}{64 \pi G l}, \quad T_{p}\left(\frac{3 \pi}{4}\right) \rightarrow-\frac{(\sqrt{2}-2) p}{64 \pi G l} .
$$

On the other hand, $T_{p}^{(4)}(40)$ is proportional to the square of $p$ :

$$
T_{p}^{(4)}\left(y_{0}\right) \rightarrow \frac{p^{2}}{64 \pi G^{(4)} l^{2} \sin ^{2} y_{0}} .
$$

Then the short distance behavior seems to be changed drastically. Eq.(52) from the pure 4d theory tells that the graviton correlator behaves as

$$
\left\langle h_{i j}(x) h_{i^{\prime} j^{\prime}}\left(x^{\prime}\right)\right\rangle \sim \int \frac{d^{4} p}{p^{2}} \mathrm{e}^{i p \cdot\left(x-x^{\prime}\right)} \sim \frac{1}{\left|x-x^{\prime}\right|^{2}},
$$

which is consistent with the Coulomb-like behavior in 4 dimensions. On the other hand, Eq.(51) gives

$$
\left\langle h_{i j}(x) h_{i^{\prime} j^{\prime}}\left(x^{\prime}\right)\right\rangle \sim \int \frac{d^{4} p}{p} \mathrm{e}^{i p \cdot\left(x-x^{\prime}\right)} \sim \frac{1}{\left|x-x^{\prime}\right|^{3}} .
$$

The behavior of $\left|x-x^{\prime}\right|^{-3}$ is nothing but the 5-dimensional one. Therefore the potential of the gravity behaves as $\frac{1}{r^{2}}$ rather than $\frac{1}{r}$. Then the gravity force in the induced gravity becomes stronger at short distances than that in the pure $4 \mathrm{~d}$ one. This indicates that one can observe the bulk 5 dimensional space effects from the short distance behavior of the correlator (36).

As a variation, we consider the case that the bulk space is anti-de Sitter space by replacing $l^{2}$ in $S_{\mathrm{EH}}$ (1). If we choose $S_{1}$ as in (1), however, there is no solution describing the $\mathrm{S}_{4}$ brane without adding the conformal anomaly induced action. Here as a toy model, we choose the tension of the brane to be arbitrary and replace $l$ by another parameter $l^{\prime}$ in $S_{1}$. Then one starts from the following action:

$$
\begin{aligned}
S & =S_{\mathrm{EH}}+S_{\mathrm{GH}}+S_{1}, \quad S_{\mathrm{EH}}=-\frac{1}{16 \pi G} \int d^{5} x \sqrt{g}\left(R+\frac{12}{l^{2}}\right), \\
S_{\mathrm{GH}} & =-\frac{1}{8 \pi G} \int d^{4} x \sqrt{\gamma} K, \quad S_{1}=\frac{3}{8 \pi G l^{\prime}} \int d^{4} x \sqrt{\gamma} .
\end{aligned}
$$


We now choose the metric of the bulk AdS (hyperboloid) as in (30). If there is a brane at $y=y_{0}$, by defining the radius of $\mathrm{S}_{4}$ by $R_{b}=l \sinh y_{0}$, we obtain the equation corresponding to (11) in the following form:

$$
\frac{1}{R_{b}} \sqrt{1+\frac{R_{b}^{2}}{l^{2}}}=\frac{1}{l^{\prime}}
$$

When $R_{b} \rightarrow+\infty$, the r.h.s. of (56) goes to $\frac{1}{l}$ and when $R_{b} \rightarrow 0$, the r.h.s. goes to the positive infinity. Then if

$$
l^{\prime}<l
$$

there is a unique non-trivial solution. The equation corresponding to (35) has the folowing form:

$$
T_{p}\left(y_{0}\right)=\frac{l^{3}}{2 \pi G} \frac{1}{32 l^{4}}\left(\frac{f_{p}^{\operatorname{AdS}^{\prime}}\left(y_{0}\right)}{f_{p}^{\operatorname{AdS}}\left(y_{0}\right)}+4 \operatorname{coth} y_{0}-\frac{6 l}{l^{\prime}}\right) .
$$

Here $f_{p}^{\mathrm{AdS}}\left(y_{0}\right)$ is defined by (32). Then the large $p$-behavior is similar to (51):

$$
T_{p}\left(y_{0}\right) \rightarrow \frac{p}{64 \pi G l} \frac{\cosh y_{0}+1}{\sinh y_{0}} .
$$

The potential of the gravity behaves as $\frac{1}{r^{2}}$ rather than $\frac{1}{r}$ at the short distance, again. Then the gravity force in the induced gravity becomes stronger at short distances than that in the pure $4 \mathrm{~d}$ one. Thus, we demonstrated that qualitative behaviour of $4 \mathrm{~d}$ induced gravity obtained from dS or AdS bulk is similar, but it differs from the standard 4d gravity behaviour.

Note that in order to obtain a behavior of the graviton correlator similar to [3], the radius of the brane should be much larger than the length parameter of the bulk de Sitter space. If we consider the Euclidean bulk, the radius is always smaller than the length parameter. Then one needs to continue analytically the bulk metric to Lorentzian. The related interesting question is about the dependence of our results from the brane tension. Imagine that we do not fix the boundary counterterm and start with the following action, instead of (1),

$$
\begin{aligned}
S & =S_{\mathrm{EH}}+S_{\mathrm{GH}}+S_{1}, \quad S_{\mathrm{EH}}=-\frac{1}{16 \pi G} \int d^{5} x \sqrt{-g}\left(R-\frac{12}{l^{2}}\right), \\
S_{\mathrm{GH}} & =-\frac{1}{8 \pi G} \int d^{4} x \sqrt{\gamma}, \quad S_{1}=-\frac{3}{8 \pi G l^{\prime}} \int d^{4} x \sqrt{\gamma} .
\end{aligned}
$$

Here we choose the coefficient of $S_{1}$ to be arbitrary, by replacing $l$ in $S_{1}$ (11) by $l^{\prime}$. Then we obtain the 5-dimensional Lorentzian de Sitter space:

$$
\begin{aligned}
d s_{\mathrm{dS}_{5}}^{2} & =l^{2}\left(-d y^{2}+\gamma_{i j} d x^{i} d x^{j}\right) \\
\gamma_{i j} & =\cosh ^{2} y \hat{\gamma}_{i j}
\end{aligned}
$$

which can be obtained from (3) by analytically continuing $y$ as 


$$
y \rightarrow \frac{\pi}{2}+i y
$$

Then the equation corresponding to (10) has the following form:

$$
\left.\partial_{y} A\right|_{\text {onthebrane }}=\frac{l}{l^{\prime}} .
$$

Here $A$ is given by

$$
A=\ln \cosh y-\ln \cosh \sigma .
$$

Defining the radius $R_{b}$ of the brane by $R_{b}=l \cosh y_{0}$, and assuming that there is a brane at $y=y_{0}$, Eq. 63) can be rewritten by

$$
\frac{1}{R_{b}} \sqrt{\frac{R_{b}^{2}}{l^{2}}-1}=\frac{l}{l^{\prime}} .
$$

Eq.(65) has a solution labeled by $l^{\prime}$ if $\frac{l}{l^{\prime}}<1$ and when $\frac{l}{l^{\prime}} \rightarrow 1-0, R_{b}$ goes to infinity.

Then the graviton correlator may be calculated as following

$$
\begin{aligned}
\left\langle h_{i j}(x) h_{i^{\prime} j^{\prime}}\left(x^{\prime}\right)\right\rangle & =\sum_{p=2}^{\infty} W_{i j i^{\prime} j^{\prime}}^{(p)}\left(x, x^{\prime}\right) \frac{1}{2 T_{p}\left(y_{0}\right)}, \\
T_{p}\left(y_{0}\right) & =-\frac{l^{3}}{2 \pi G} \frac{1}{32 l^{4}}\left(\frac{f_{p}^{\prime}\left(y_{0}\right)}{f_{p}\left(y_{0}\right)}+4 \tanh y_{0}-\frac{6 l}{l^{\prime}}\right), \\
f_{p}(y) & \propto(\cosh y)^{1 / 2}\left(P_{-5 / 2}^{-(p+3 / 2)}(i \sinh y)+P_{-5 / 2}^{-(p+3 / 2)}(-i \sinh y)\right) .
\end{aligned}
$$

We now consider the case that the radius of the brane $R_{b}$ is large, then $y_{0}$ is also large and $\frac{l}{l^{\prime}} \rightarrow 1-0$. Since when $R_{b}$ is large

$$
\frac{f_{p}^{\prime}\left(y_{0}\right)}{f_{p}\left(y_{0}\right)} \rightarrow 2-\frac{l^{2}}{2 R_{b}^{2}}(p+1)(p+2)+\mathcal{O}\left(\frac{l^{4}}{R_{b}^{4}}\right)
$$

one gets

$$
T_{p}\left(y_{0}\right) \rightarrow \frac{l}{128 \pi G R_{b}^{2}}(p+1)(p+2)
$$

The corresponding equation in the pure $4 \mathrm{~d}$ gravity is given in (40) replacing $l \sin y_{0}$ by $R_{b}$ :

$$
T_{p}^{(4)}\left(y_{0}\right)=-\frac{1}{128 \pi G^{(4)} R_{b}^{2}}\left\{(2-p(p+3))-R_{b}^{2} \Lambda^{(4)}-8\right\} .
$$

Then if we choose

$$
G^{(4)}=\frac{G}{l}, \quad \Lambda^{(4)}=-\frac{9}{R_{b}^{2}},
$$

we find $T_{p}\left(y_{0}\right) \rightarrow T_{p}^{(4)}\left(y_{0}\right)$ when the brane is large $\left(R_{b} \rightarrow \infty\right.$ or $\left.\frac{l}{l^{\prime}} \rightarrow 1-0\right)$. Thus, in this limit the induced gravity coincides with the pure $4 \mathrm{~d}$ gravity where the Newton constant $G^{(4)}$ and the cosmological constant $\Lambda^{(4)}$ is given by $(70)$. We should note again that the brane can exist only when $l^{\prime}<l$.

Note that in the correlation function $\left\langle h_{i j}(x) h_{i^{\prime} j^{\prime}}\left(x^{\prime}\right)\right\rangle$ it is assumed $l, l^{\prime} \ll\left|x-x^{\prime}\right| \ll R_{b}$. That is, the distance $\left|x-x^{\prime}\right|$ is much shorter than the radius of the brane $R_{b}$ but much larger than $l$ and $l^{\prime}$. At very short distance $\left|x-x^{\prime}\right| \ll l, l^{\prime}$, the correlation function behaves as 5-dimensional one as in 54. 


\section{GRAVITON CORRELATOR AND ACTION WITH ACCOUNT OF CONFORMAL QUANTUM MATTER}

Let us try to analyze the effect of quantum CFT to graviton correlator. As shown in (51) and (59), $T_{p}\left(y_{0}\right)$ in the induced gravity behaves linearly in $p$ for large $p$ and the graviton correlator shows its 5-dimensional structure at short distances. As pointed out in [3, 10], however, the short distance behavior is changed by including the quantum matter fields on the brane (by adding the effective action $\mathcal{W}$, which is induced from the conformal anomaly of the matter fields, to the action (1)):

$$
\begin{aligned}
\mathcal{W}= & -b \int d^{4} x \sqrt{\widetilde{\gamma}} \widetilde{F} A-b^{\prime} \int d^{4} x \sqrt{\widetilde{\gamma}}\left\{A \left[2 \widetilde{\square}^{2}+\widetilde{R}_{\mu \nu} \widetilde{\nabla}_{\mu} \widetilde{\nabla}_{\nu}-\frac{4}{3} \widetilde{R}_{\square^{2}}^{2}\right.\right. \\
& \left.\left.+\frac{2}{3}\left(\widetilde{\nabla}^{\mu} \widetilde{R}\right) \widetilde{\nabla}{ }_{\mu}\right] A+\left(\widetilde{G}-\frac{2}{3} \widetilde{\square} \widetilde{R}\right) A\right\} \\
& +\frac{1}{12}\left\{b^{\prime \prime}+\frac{2}{3}\left(b+b^{\prime}\right)\right\} \int d^{4} x \sqrt{\widetilde{\gamma}}\left[\widetilde{R}-6 \widetilde{\square}^{A}-6\left(\widetilde{\nabla}_{\mu} A\right)\left(\widetilde{\nabla}^{\mu} A\right)\right]^{2} .
\end{aligned}
$$

Here one chooses the 4-dimensional boundary metric as

$$
\gamma_{i j}=\mathrm{e}^{2 A} \tilde{\gamma}_{i j}
$$

and we specify the quantities with $\tilde{\gamma}_{i j}$ by using $\sim G(\tilde{G})$ and $F(\tilde{F})$ are the Gauss-Bonnet invariant and the square of the Weyl tensor, which are given as

$$
G=R^{2}-4 R_{i j} R^{i j}+R_{i j k l} R^{i j k l}, \quad F=\frac{1}{3} R^{2}-2 R_{i j} R^{i j}+R_{i j k l} R^{i j k l},
$$

In the effective action (71) induced by brane quantum matter, in general, with $N$ real scalar, $N_{1 / 2}$ Dirac spinor, $N_{1}$ vector fields, $N_{2}(=0$ or 1$)$ gravitons and $N_{\mathrm{HD}}$ higher derivative conformal scalars, $b, b^{\prime}$ and $b^{\prime \prime}$ are

$$
\begin{aligned}
& b=\frac{N+6 N_{1 / 2}+12 N_{1}+611 N_{2}-8 N_{\mathrm{HD}}}{120(4 \pi)^{2}}, \\
& b^{\prime}=-\frac{N+11 N_{1 / 2}+62 N_{1}+1411 N_{2}-28 N_{\mathrm{HD}}}{360(4 \pi)^{2}}, \quad b^{\prime \prime}=0 .
\end{aligned}
$$

Usually, $b^{\prime \prime}$ may be changed by the finite renormalization of local counterterm in the gravitational effective action but this parameter plays an important role in tensor perturbations, which leads to decay of de Sitter space (end of inflation) [10].

On the brane, we can choose $h_{i}^{i}=0$ as one of the gauge conditions. Then since the area of the brane is invariant under scale transformation, one can regard that the scale factor $A(x)$ is constant, $A(x)=A_{0}$ on the brane. Under this condition, the eq.(71) becomes as

$$
\begin{aligned}
\mathcal{W}= & -b \int d^{4} x \sqrt{\widetilde{\gamma}}\left(-\frac{2}{3} \widetilde{R}^{2}+2 \widetilde{R}_{i j} \widetilde{R}^{i j}\right) A_{0} \\
& +\frac{1}{12}\left\{b^{\prime \prime}+\frac{2}{3}\left(b+b^{\prime}\right)\right\} \int d^{4} x \sqrt{\widetilde{\gamma}} \widetilde{R}^{2} \\
& =-b \int d^{4} x \sqrt{\gamma}\left(-\frac{2}{3} R^{2}+2 R_{i j} R^{i j}\right) A_{0} \\
& +\frac{1}{12}\left\{b^{\prime \prime}+\frac{2}{3}\left(b+b^{\prime}\right)\right\} \int d^{4} x \sqrt{\gamma} R^{2} .
\end{aligned}
$$


Here we use the relation $\sqrt{\widetilde{\gamma}}=e^{-4 A_{0}} \sqrt{\gamma}$ and $\widetilde{R}=e^{2 A_{0}} R, \widetilde{R}_{i j}=R_{i j}$.

$$
\begin{aligned}
R & =R_{(0)}+\frac{1}{2} \delta^{2} R+\mathcal{O}\left(h^{3}\right), \\
\delta^{2} R & \equiv \frac{2}{\tilde{l}^{2}} h_{i j} h^{i j}+\frac{1}{2} h^{i j} \nabla^{2} h_{i j}+\nabla^{2}\left(h_{i j} h^{i j}\right), \\
\sqrt{\gamma} & =\sqrt{\gamma_{(0)}}\left(1-\frac{1}{4} h^{i j} h_{i j}+\mathcal{O}\left(h^{3}\right)\right) .
\end{aligned}
$$

Here $\tilde{l}=R_{b}=l \sinh y_{0}\left(\sin y_{0}\right)$ when the bulk 5 dimensional space is anti-deSitter (de Sitter). Then neglecting $\mathcal{O}\left(h^{3}\right)$ terms, one finds

$$
\begin{aligned}
\int d^{4} x \sqrt{\gamma} R^{2}=\int & d^{4} x \sqrt{\gamma}\left\{R_{(0)}^{2}-\frac{1}{4} h^{i j} h_{i j} R_{(0)}^{2}\right. \\
& \left.+\left(\frac{2}{\tilde{l}^{2}} h_{i j} h^{i j}+\frac{1}{2} h^{i j} \nabla^{2} h_{i j}+\nabla^{2}\left(h_{i j} h^{i j}\right)\right) R_{(0)}\right\} \\
= & \int d^{4} x \sqrt{\gamma_{(0)}}\left\{\frac{144}{\tilde{l}^{4}}-\frac{12}{\tilde{l}^{4}} h_{i j} h^{i j}+\frac{6}{\tilde{l}^{2}} h^{i j} \nabla^{2} h_{i j}+\frac{12}{\tilde{l}^{2}} \nabla^{2}\left(h_{i j} h^{i j}\right)\right\} .
\end{aligned}
$$

Expanding the Ricci tensor $R_{i j}$ with respect to $h_{i j}$

$$
R_{i j}=R_{(0) i j}+\delta R_{i j}+\frac{1}{2} \delta^{2} R_{i j}+\mathcal{O}\left(h^{3}\right)
$$

and using following expressions,

$$
\begin{aligned}
\delta R_{i j} & =\frac{1}{2}\left(\frac{8}{\tilde{l}^{2}} h_{i j}-\nabla^{2} h_{i j}\right) \\
\gamma^{i j} \delta^{2} R_{i j} & =\frac{4}{\tilde{l}^{2}} h_{i j} h^{i j}+\nabla^{2}\left(h_{i j} h^{i j}\right)-\frac{1}{2} h^{i j} \nabla^{2} h_{i j},
\end{aligned}
$$

we obtain

$$
\begin{array}{rl}
\int d^{4} & x \sqrt{\gamma} R_{i j} R_{k l} \gamma^{k i} \gamma^{l j}=\int d^{4} x \sqrt{\gamma}\left\{R_{(0) i j} R_{(0) k l} \gamma_{(0)}^{k i} \gamma_{(0)}^{l j}\right. \\
& +2 \delta R_{i j} R_{(0) k l} \gamma_{(0)}^{k i} \gamma_{(0)}^{l j}-2 R_{(0) i j} R_{(0) k l} \gamma_{(0)}^{k n} h_{n m} \gamma_{(0)}^{m i} \gamma_{(0)}^{l j} \\
& +\delta^{2} R_{i j} R_{(0) k l} \gamma_{(0)}^{k i} \gamma_{(0)}^{l j} \\
& +2 R_{(0) i j} R_{(0) k l} \gamma_{(0)}^{k n} h_{n m} \gamma_{(0)}^{m \tau} h_{\tau \rho} \gamma_{(0)}^{\rho i} \gamma_{(0)}^{l j} \\
& -4 \delta R_{i j} R_{(0) k l} \gamma_{(0)}^{k m} h_{m n} \gamma_{(0)}^{n i} \gamma_{(0)}^{l j}+\delta R_{i j} \delta R_{k l} \gamma_{(0)}^{k i} \gamma_{(0)}^{l j} \\
& \left.+R_{(0) i j} R_{(0) k l} \gamma_{(0)}^{k n} h_{n m} \gamma_{(0)}^{m i} \gamma_{(0)}^{l \tau} h_{\tau \lambda} \gamma_{(0)}^{\lambda j}\right\} \\
= & \int d^{4} x \sqrt{\gamma_{(0)}}\left\{\frac{36}{\tilde{l}^{4}}-\frac{2}{\tilde{l}^{4}} h^{i j} h_{i j}+\frac{1}{2 \tilde{l}^{2}} h^{i j} \nabla^{2} h_{i j}\right. \\
& \left.+\frac{3}{\tilde{l}^{2}} \nabla^{2}\left(h_{i j} h^{i j}\right)+\frac{1}{4}\left(\nabla^{2} h_{i j}\right)^{2}\right\} .
\end{array}
$$

Substituting eqs.(77) and (80) into eq.(75) gives : 


$$
\begin{aligned}
\mathcal{W}= & \left\{\frac{2}{3} b\left(\frac{1}{12}+A_{0}\right)+\frac{1}{12}\left(b^{\prime \prime}+\frac{2}{3} b^{\prime}\right)\right\} \int d^{4} x \sqrt{\gamma} R^{2} \\
& -2 b A_{0} \int d^{4} x \sqrt{\gamma} R_{i j} R^{i j} \\
= & \left\{\frac{2}{3} b\left(\frac{1}{12}+A_{0}\right)+\frac{1}{12}\left(b^{\prime \prime}+\frac{2}{3} b^{\prime}\right)\right\} \int d^{4} x \sqrt{\gamma(0)}\left\{\frac{144}{\tilde{l}^{4}}-\frac{12}{\tilde{l}^{4}} h_{i j} h^{i j}\right. \\
+ & \left.\frac{6}{\tilde{l}^{2}} h^{i j} \nabla^{2} h_{i j}+\frac{12}{\tilde{l}^{2}} \nabla^{2}\left(h_{i j} h^{i j}\right)\right\}-2 b A_{0} \int d^{4} x \sqrt{\gamma(0)}\left\{\frac{36}{\tilde{l}^{4}}-\frac{2}{\tilde{l}^{4}} h^{i j} h_{i j}\right. \\
+ & \left.\frac{1}{2 \tilde{l}^{2}} h^{i j} \nabla^{2} h_{i j}+\frac{3}{\tilde{l}^{2}} \nabla^{2}\left(h_{i j} h^{i j}\right)+\frac{1}{4}\left(\nabla^{2} h_{i j}\right)^{2}\right\} \\
= & \int d^{4} x \sqrt{\gamma(0)}\left[\left\{b\left(\frac{1}{18}+\frac{1}{6} A_{0}\right)+\frac{1}{12}\left(b^{\prime \prime}+\frac{2}{3} b^{\prime}\right)\right\} \frac{144}{\tilde{l}^{4}}\right. \\
& -\left\{b\left(\frac{1}{18}+\frac{1}{3} A_{0}\right)+\frac{1}{12}\left(b^{\prime \prime}+\frac{2}{3} b^{\prime}\right)\right\} \frac{12}{\tilde{l}^{4}} h_{i j} h^{i j} \\
+ & \left\{b\left(\frac{1}{18}+\frac{1}{2} A_{0}\right)+\frac{1}{12}\left(b^{\prime \prime}+\frac{2}{3} b^{\prime}\right)\right\} \frac{6}{\tilde{l}^{2}} h^{i j} \nabla^{2} h_{i j} \\
+ & \left.\left\{b\left(\frac{1}{18}+\frac{1}{6} A_{0}\right)+\frac{1}{12}\left(b^{\prime \prime}+\frac{2}{3} b^{\prime}\right)\right\} \frac{12}{\tilde{l}^{2}} \nabla^{2}\left(h_{i j} h^{i j}\right)-\frac{1}{2} b A_{0}\left(\nabla^{2} h_{i j}\right)^{2}\right] .
\end{aligned}
$$

Using (20), one finds the last term $-\frac{1}{2} b A_{0}\left(\nabla^{2} h_{i j}\right)^{2}$ gives the leading contribution at short distances (that is, large $p$ ) as $\left(\nabla^{2} h_{i j}\right)^{2} \sim p^{4} h_{i j} h^{i j}$. Then we find that, by including the effective action $\mathcal{W}$ in (71), the term proportional to $p^{4}$ appears in $T_{p}\left(y_{0}\right)$ (37) or $T_{p}^{(4)}\left(y_{0}\right)$ (40). Then the short distance fluctuation of $h_{i j}$ is highly suppressed and the graviton correlator behaves as

$$
\left\langle h_{i j}(x) h_{i^{\prime} j^{\prime}}\left(x^{\prime}\right)\right\rangle \sim \int \frac{d^{4} p}{p^{4}} \mathrm{e}^{i p \cdot\left(x-x^{\prime}\right)} \sim \ln \left|x-x^{\prime}\right| .
$$

In the pure $4 \mathrm{~d}$ theory, the total action is given by a sum of the action (39) and $\mathcal{W}(81)$

$$
S_{\text {total }}^{(4)}=S_{\mathrm{EH}}^{(4)}+\mathcal{W}
$$

On the other hand, the total action of the $4 \mathrm{~d}$ gravity induced from $5 \mathrm{~d}$ is the sum of the action (34) and $\mathcal{W}$ :

$$
S_{\text {total }}=S_{\text {correlator }}+\mathcal{W}
$$

In both cases, $\mathcal{W}$ gives the dominant contribution $p^{4}$ at short distances (large $p$ ) and the pure graviton contribution $p^{2}$ or $p$ is always suppressed by adding $\mathcal{W}$. Then there does not appear the difference between the short distance behaviours of the graviton correlator from the pure 4 dimensional gravity (39) and that of the gravity induced from 5 dimensional gravity.

In [3], the graviton correlator has been found with the help of the higher order surface counterterms, which include the square of the curvatures. The counterterms are related with the conformal anomaly of the matter on the brane (in frames of AdS/CFT correspondence). Then, when the radius of the $4 \mathrm{~d}$ de Sitter brane is large, it has been found the following graviton correlator in case that the matter is $\mathcal{N}=4 S U(N)$ or $U(N)$ super Yang-Mills: 


$$
\begin{aligned}
\left\langle h_{i j}(x) h_{i^{\prime} j^{\prime}}\left(x^{\prime}\right)\right\rangle & =\sum_{p=2}^{\infty} W_{i j^{\prime} j^{\prime}}^{(p)}\left(x, x^{\prime}\right) \frac{1}{2 T_{p}^{\mathrm{HHR}}\left(y_{0}\right)}, \\
T_{p}^{\mathrm{HHR}}\left(y_{0}\right) & =\frac{1}{8 N^{2} G_{4}^{2}}\left(p^{2}+3 p+6+\Psi(p)\right) .
\end{aligned}
$$

Here $G_{4}$ is the 4 dimensional Newton constant and $\Psi(p)$ is given by

$$
\begin{aligned}
\Psi(p)= & p(p+1)(p+2)(p+3)\left[\psi\left(\frac{p+5}{2}\right)+\psi\left(\frac{p+4}{2}\right)-\psi(2)-\psi(1)\right] \\
& +p^{4}+2 p^{3}-5 p^{2}-10 p-6
\end{aligned}
$$

with $\psi(z) \equiv \frac{\Gamma^{\prime}(z)}{\Gamma(z)}$. Then $T_{p}^{\mathrm{HHR}}\left(y_{0}\right)$ behaves as $p^{4} \ln p$ for large $p$ [10]. Neglecting $\ln p$, the behavior seems to be consistent with the results given in this section. The formulation is, however, valid for the case that the matter on the brane is $\mathcal{N}=4 S U(N)$ or $U(N)$ Yang-Mills theory since the higher order counterterms are used in [3,10] in order to evaluate the quantum contribution from CFT on the brane. The higher order counterterms can be determined uniquely from the geometry of the bulk AdS, which does not distinguish what kind of matter exists on the brane. Then the corresponding brane CFT should be always $\mathcal{N}=4 S U(N)$ or $U(N)$ Yang-Mills theory. Since we used the conformal anomaly induced action in order to evaluate the contribution from the quantum effects, the formulation in this paper can be applied for any (conformal) matter theory.

\section{DISCUSSION}

In summary, we calculated the graviton correlator in the brane-world model with fixed brane tension where bulk represents dS space and the brane is (inflationary) de Sitter Universe. It is shown that like in the standard AdS/CFT related brane-worlds the short distance behaviour of the graviton correlator differs from that in General Relativity. However, account of quantum effects of CFT living on the brane drastically changes the short distance behaviour of correlator.

In refs. [0,2 the quantum induced brane, which is the 4 dimensional sphere in 5dimensional anti-de Sitter space, has been considered. In order for such a brane to exist one needs the conformal anomaly induced action (71). As a result the obtained correlator behaves as (82) unlike to the case of pure graviton correlator (54).

The short distance behavior (53) of the induced gravity is different from that of the pure Einstein gravity (54) if we do not include the quantum effects of the matter on the brane. Eq.(82) tells, however, the quantum effects become dominant at short distances and smear the difference. Furthermore the quantum effects strongly suppress the fluctuations of the graviton at short distances. In other words, the quantum effects make the spacetime very rigid on small scales. This is universal feature of quantum CFT whatever the gravitational part is. Our discussion gives further support for consideration of dS brane-worlds on equal footing with AdS brane-worlds. 


\section{ACKNOWLEDGMENTS}

The work by S.N. is supported in part by the Ministry of Education, Science, Sports and Culture of Japan under the grant n. 13135208. The work by S.O. is supported in part by the Japan Society for the Promotion of Science under the Postdoctoral Research Program. 


\section{REFERENCES}

[1] J. Louko and D. Wiltshire, JHEP 0202 (2002) 007, hep-th/0109099.

[2] S. Nojiri, S.D. Odintsov and S. Zerbini, Phys.Rev. D62 (2000) 064006, hep-th/0001192;

S. Nojiri and S.D. Odintsov, Phys.Lett. B484 (2000) 119, hep-th/0004097; Gravitation and Cosmology 8 (2002), hep-th/0105160.

[3] S.W. Hawking, T. Hertog and H.S. Reall, Phys. Rev. D62 (2000) 043501, hepth/0003052.

[4] M. Spradlin, A. Strominger and A. Volovich, hep-th/0110007.

[5] S. Nojiri, S.D. Odintsov, JHEP 0112 (2001) 033, hep-th/0107134.

[6] J. de Boer, E. Verlinde and H. Verlinde,JHEP 08003 (2000), hep-th/9912012; E. Verlinde and H. Verlinde, JHEP 05034 (2000), hep-th/9912018.

[7] S. Nojiri and S.D. Odintsov, Phys.Lett. B519 (2001) 145, hep-th/0106191.

[8] B. Allen and T. Jacobson, Comm. Math.Phys. 103669 (1986); A. Higuchi, J. Math.Phys. 281553 (1987); B. Allen and M. Turyn, Nucl.Phys. B292 813 (1987); K. Yamamoto, M. Sasaki and T. Tanaka, Phys.Rev. D54 5031 (1996); H. Osborn and G. Shore, hep-th/9909043.

[9] L. Randall and R. Sundrum, Phys.Rev.Lett. 833370 (1999), hep-th/9905221; Phys.Rev.Lett. 834690 (1999), hep-th/9906064.

[10] S.W. Hawking, T. Hertog and H.S. Reall, Phys.Rev. D63 (2001) 083504; hepth/0010232. 